\title{
Modified radical mastectomy for anterior thoracic nerve and intercostobrachial nerve protection (case report)
}

\author{
Shengchao Huang", Pu Qiü, Weizhang Chen, Yuanqi Zhang, Kangwei Luo, Jianwen Li \\ Department of Breast Surgery, Affiliated Hospital of Guangdong Medical University, Zhanjiang 524001, China \\ \#These authors contributed equally to this work. \\ Correspondence to: Jianwen Li; Yuanqi Zhang. Department of Breast Surgery, Affiliated Hospital of Guangdong Medical University, Zhanjiang 524001, \\ China. Email: 982688169@qq.com; 513614508@qq.com.
}

\begin{abstract}
Modified breast cancer radical mastectomy is a more common operating method in breast surgery. Traditional modified radical mastectomy focuses on protecting the long thoracic nerve and thoracodorsal nerve while ignoring the protection of the anterior thoracic nerve and intercostobrachial nerve protection, which leads often to patients with upper medial arm numbness, acid swelling, pain, chest atrophy, and other problems. In the modified radical mastectomy of breast cancer, in this case, the author used an elaborative operation to protect the anterior thoracic nerve and intercostobrachial nerve and thoroughly dissected the third-level lymph nodes through the axillary approach.
\end{abstract}

Keywords: Breast cancer; anterior thoracic nerve; intercostobrachial nerve

Submitted Dec 18, 2019. Accepted for publication Feb 17, 2020.

doi: $10.21037 /$ gs.2020.02.17

View this article at: http://dx.doi.org/10.21037/gs.2020.02.17

\section{Introduction}

Breast cancer is the most common malignant tumor in women (1). Breast cancer is a disease that requires systemic treatment. Surgery, however, is still an effective way of treating breast cancer (2). Nowadays, precision treatment is the general trend of medical treatment. In surgery, our surgeons can achieve precisely and improve the surgery methods based on eradicating tumors, reducing the scope of surgical incisions, and reducing unnecessary injuries during surgery (3).

Nevertheless, most hospitals are all adopting traditional modified radical mastectomy. Their treatment stresses anterior thoracic nerve and intercostobrachial nerve protection which pay no enough attention to clinical significance for the anterior thoracic nerve and intercostobrachial nerve that retain only pure sensory functions (4). In the modified radical mastectomy of breast cancer, in this case, the author used an elaborative operation to protect the anterior thoracic nerve and intercostobrachial nerve, and thoroughly dissected the third-level lymph nodes through the axillary approach. We present the following case following the CARE Guideline.

\section{Condition profile}

Patient, female, 41 years old. The main complaint was discovering a left breast mass after five months. The patient had unintentionally found a left breast mass of about $1.5 \mathrm{~cm}$ $\times 1.0 \mathrm{~cm} 5$ months ago, with a hard texture, good activity, no pain, no nipple discharge, and no treatment was performed. There is no special history, personal history, and family history. College of physical: a mass of about $2.3 \mathrm{~cm} \times 1.5 \mathrm{~cm}$ can be reached and located in the 5-point direction of the left breast away from the nipple about $2.5 \mathrm{~cm}$. The mass is hard, the border is unclear, and the mobility is acceptable. At the left breast 6-point direction away from the nipple about $3 \mathrm{~cm}$, a tumor can be reached with a size of about $1.0 \mathrm{~cm} \times 1.0 \mathrm{~cm}$, which is hard, with unclear boundaries, and its mobility is acceptable. No dimple sign or orange peel sign. The left axillary can touch the swollen lymph nodes without fusion. The left supraclavicular area did not reach swollen lymph nodes. 
* Color Doppler ultrasound (breast): a hypoechoic nodule is visible at 5 o'clock on the left breast, approximately $2.3 \mathrm{~cm} \times 1.5 \mathrm{~cm}$ in size, a hypoechoic nodule, approximately $1.0 \mathrm{~cm} \times 1.0 \mathrm{~cm}$ in size, can be seen at 6 o'clock on the left breast (BI-RADS category 5). Swollen lymph nodes were found in the left axillary cavity with poor structure. Swollen lymph nodes were found under the left clavicle with a regular structure. No swollen lymph nodes were found in the left supraclavicular region.

* Molybdenum target: dense calcification area (BIRADS Category 4C) is visible under the left breast.

* CT Scan-chest: no metastatic lesions were seen in the lungs and liver.

* Whole-body bone scan: no signs of metastasis were seen in the whole-body bone.

* Hollow needle puncture pathology: left breast invasive carcinoma (grade II), ER (70\%, 3+), PR (40\%, 3+), Her-2 (1+), Ki-67 (10\%, 1+).

* Fine needle aspiration of the left axillary lymph node: cancer cells are visible.

- Postoperative pathology: non-special invasive carcinoma of the left breast (grade II), with ductal carcinoma in situ, can be diagnosed and its largest lesion diameter is about $2.2 \mathrm{~cm}$. No cancer is found in the basal and skin margins; cancer metastasis was seen in the left axillary I level lymph nodes (4/19), cancer metastasis was found in lymph nodes at level II (1/3), no cancer metastasis was found in lymph nodes at level III (0/2), and no cancer metastasis was found in the intrathoracic tissue.

* Preoperative diagnosis: left breast cancer (cT2N1M0, stage IIb, Luminal type A).

- Postoperative diagnosis: left breast invasive carcinoma (pT2N2M0, stage IIIA, Luminal type A).

* Surgery: modified radical mastectomy for left breast cancer. After surgery, chemotherapy was given (EC $\times 4 \rightarrow \mathrm{T} \times 4$ ), standard-dose radiotherapy was given, and OFS + AI endocrine therapy was given (5).

\section{Surgical skills}

The tumor is found at 5 and 6 o'clock in the left breast. An oblique incision is designed to remove the skin and puncture needles pinhole on the surface of the tumor. Use a scalpel to cut the skin and an electro tome to cut the subcutaneous tissue. At the same time, the assistant uses straight forceps to clamp the dermis and pull the flap upwards. The surgeon gently pulls the breast with a blood pad. Next, the surgeon separates the flaps by electro tome in the superficial layer of the superficial fascia of the breast without vascular space, paying attention to protect the vascular network of the flap. The separation range of the flaps reaches the predefined boundary of the preoperative line drawing: up to the lower edge of the clavicle, down to the upper edge of the rectus abdominis, inward to the side of the sternum, and outward to the front edge of the latissimus dorsi. Use the forceps to lift the upper edge of the breast, use the electro tome to perform anatomy following the direction of the pectoralis fibers on the deep side of the pectoralis fascia, and completely remove the breast tissue. While encountering the thick perforator vessels in the second, third intercostal, and anterior diastolic muscle. The electro tome is used for coagulation and hemostasis. Separate along the pectoralis major and pectoralis minor lateral edges to find the thoracic branch of the lateral thoracic blood vessels, dissect along the lateral thoracic blood vessels to the axilla, cut open the coracoclavicular fascia, expose tongue-like fat and axillary veins, and use the ultrasonic knife to cut off and coagulate the anterior muscle branch and the mammary branch of the lateral thoracic vessels. One assistant uses the deep pull hook to pull the pectoralis major and pectoralis minor apart; another lifts the disinfected limb, and the surgeon cleans the three-level and two-level lymph nodes. After cleaning the three-level and two-level lymph nodes, the surgeon can manage the first-level lymph nodes easily. First, two thicker intercostobrachial nerves will be separated and protected; secondly, the axillary tissue is pulled outward to reveal and protect long thoracic nerve; lastly, clean the lymph nodes in the inner triangle. Separate and ligate the upper thorax vein, revealing the protection of the subscapular blood vessels, the thoracodorsal nerve, and the medial antebrachial cutaneous nerve. Then, the surgeon cleans the lymph nodes in the outer triangle and completely removes the axillary lymph nodes and breast specimens. While the assistant pulls the pectoralis major muscles upwards with a hook, the surgeon can clean the lymph nodes between the pectoralis muscles, and pay attention to protect the anterior thoracic nerve, the thoracic branch of thoracic acromial vessels, and the thoracic branch of lateral thoracic vessels. Examine the degree of axillary lymph nodes for dissection and active bleeding thoroughly, rinse the wound with sterile distilled water, and place a negative pressure drainage tube in the axillary and chest wall. In the end, suture the subcutaneous and intradermal tissues with absorbable threads (Figure 1). 

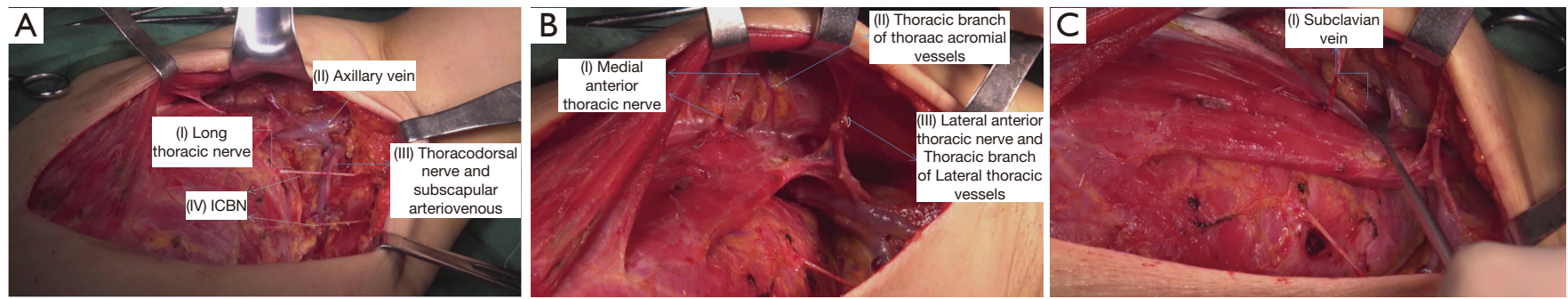

Figure 1 Originated from Department of Breast surgery of Guangdong Medical university, used and published in case reports with the consent of patients. (A) Structure of the armpit after dissection: (I) long thoracic nerve; (II) axillary vein; (III) thoracodorsal nerve and subscapular arteriovenous; (IV) ICBN. (B) The structure between pectoral muscles after dissection: (I) medial anterior thoracic nerve; (II) thoracic branch of thoracic acromial vessels; (III) lateral anterior thoracic nerve and thoracic branch of lateral thoracic vessels. (C) Threelevel lymph nodes: subclavian vein. ICBN, intercostobrachial nerve.

\section{Discussion}

Intercostobrachial nerve often have multiple branches $(6,7)$, the thickest one consists of the posterior branch of the lateral cutaneous branch of the 2 nd intercostal nerve and the lateral cutaneous branch of the 1st and $3 \mathrm{rd}$ intercostal nerve (sometimes including the medial cutaneous nerve of the arm and presenting a y-shaped confluence with the trunk of the intercostobrachial nerve on the lateral side of the third segment of the axillary vein). The intercostobrachial nerve passes through the intercostal muscle in the 2 nd intercostal space behind the outer margin of the pectoralis minor, passes through the axillary fat pad, crosses the anterior margin of the latissimus dorsi muscle, and runs laterally. Its trunk is parallel to the axillary vein (8). In surgery, as long as we are familiar with these anatomical structures, there will be no difficulty in protecting them (9). In this case, the surgeon firstly exposes the intercostobrachial nerve at the beginning of the intercostobrachial nerve, and then along the front edge of the latissimus dorsi muscle separates the tissue attached to it to find the intercostobrachial nerve reaching the medial side of the upper arm, which is the anatomical characteristics of it. Finally, the two met. At this time, the surgeon can dissect the axillary tissue and intercostal arm nerves. When dissecting the intercostal arm nerve, it is best to dissect with the blunt classification method to avoid thermal damage of the electro tome and nerve damage caused by the excessive stretch. The anterior thoracic nerve is the main innervating nerve of the pectoralis major muscle and originates from the medial and lateral tracts of the brachial plexus (10). It is divided into the medial anterior thoracic nerve and lateral anterior thoracic nerve. The medial anterior thoracic nerve originates from the lateral brachial plexus, parallels to the pectoral branch of the thoracoacromial artery, runs on the inner side of the pectoralis minor and innervates the pectoralis major muscle. There are three types of innervation: (I) bypass the upper edge of the pectoralis minor; (II) pass through the upper $1 / 3$ and middle $1 / 3$ of the pectoralis minor; (III) bypass the lower edge of the pectoralis minor. The travel of these branches is closely related to the pectoralis minor and variation is rarely found. The lateral anterior thoracic nerve originates from the medial brachial plexus but runs to the outside of the pectoralis minor muscles. It goes with the pectoral muscle branches of the lateral pectoral vessels and innervates the outside of the pectoralis major muscle. Master the anatomical anatomy of the anterior thoracic nerve is more important for its protection of the operation. When clean Rotter's lymph nodes, Delicate surgical procedures are the key to protect the anterior thoracic nerve.

In the modified radical mastectomy, the more difficult operation is to clean the third-level lymph nodes (11). In traditional surgery, it is often necessary to open the window of the pectoral muscle or cut off the pectoralis minor to clean the third-level lymph nodes, which possibly causes greater trauma. In this case, through the lateral axillary approach, one assistant pulls the pectoralis minor and pectoralis major muscles apart with deep hooks, and the other raises the affected limb. With the anesthesiologist increasing muscle relaxation, the subclavian vein will be well exposed. This method is less invasive, reduces surgical time, and conforms to the surgical principle of lump-based resection $(12,13)$.

\section{Acknowledgments}

Funding: None. 


\section{Footnote}

Conflicts of Interest: All authors have completed the ICMJE uniform disclosure form (available at http://dx.doi. org/10.21037/gs.2020.02.17). The authors have no conflicts of interest to declare.

Ethical Statement: The authors are accountable for all aspects of the work in ensuring that questions related to the accuracy or integrity of any part of the work are appropriately investigated and resolved. Written informed consent was obtained from the patient for publication of this manuscript and any accompanying images.

Open Access Statement: This is an Open Access article distributed in accordance with the Creative Commons Attribution-NonCommercial-NoDerivs 4.0 International License (CC BY-NC-ND 4.0), which permits the noncommercial replication and distribution of the article with the strict proviso that no changes or edits are made and the original work is properly cited (including links to both the formal publication through the relevant DOI and the license). See: https://creativecommons.org/licenses/by-nc-nd/4.0/.

\section{References}

1. Torre LA, Bray F, Siegel RL, et al. Global cancer statistics, 2012. CA Cancer J Clin 2015;65:87-108.

2. Viale G, Marra A, Curigliano G, et al. Toward precision medicine in inflammatory breast cancer. Transl Cancer Res 2019;8:S469-S478.

3. Murugappan K, Saboo A, Kuo L, et al. Paradigm shift in the local treatment of breast cancer: mastectomy to breast conservation surgery. Gland Surg 2018;7:506-19.

4. Celenay ST, Ucurum SG, Kaya DO. Comparison of Spinal Alignment and Mobility in Women With and Without Post Modified Radical Mastectomy Unilateral Lymphoedema. Clin Breast Cancer 2019. doi: 10.1016/ j.clbc.2019.10.003

5. Poggio F, Ceppi M, Lambertini M, et al. Concurrent

Cite this article as: Huang S, Qiu P, Chen W, Zhang Y, Luo K, Li J. Modified radical mastectomy for anterior thoracic nerve and intercostobrachial nerve protection (case report). Gland Surg 2020;9(2):463-466. doi: 10.21037/gs.2020.02.17 versus sequential adjuvant chemo-endocrine therapy in hormone-receptor positive early stage breast cancer patients: a systematic review and meta-analysis. Breast 2017;33:104-8.

6. Zhu J, Jiao D, Guo X, et al. Predictive factors and prognostic value of pathologic complete response of ipsilateral supraclavicular lymph nodes in breast cancer after neoadjuvant chemotherapy. Ann Transl Med 2019;7:666.

7. Henry BM, Graves MJ, Pękala JR, et al. Origin, Branching, and Communications of the Intercostobrachial Nerve: a Meta-Analysis with Implications for Mastectomy and Axillary Lymph Node Dissection in Breast Cancer. Cureus 2017;9:e1101.

8. Loukas M, Louis RG Jr, Fogg QA, et al. An unusual innervation of pectoralis minor and major muscles from a branch of the intercostobrachial nerve. Clin Anat 2006;19:347-9.

9. Dasgupta S, Sanyal S, Sengupta SP. Transpectoral anterior approach to the axilla for lymph node dissection in association with mastectomy preserving both pectoral muscles and their neurovascular bundles. Tumori 1999;85:498-502.

10. Rao R, Euhus D, Mayo HG, et al. Axillary node interventions in breast cancer: a systematic review. JAMA 2013 Oct 2;310:1385-94.

11. Brun JL, Rousseau E, Belleannée G, et al. Axillary lymphadenectomy prepared by fat and lymph node suction in breast cancer. Eur J Surg Oncol 1998;24:17-20.

12. Breast Cancer Professional Committee of Chinese AntiCancer Association. Guidelines and norms for diagnosis and treatment of breast cancer of Chinese anti-cancer association (2017 edition). Chinese Journal of Cancer 2017;27:695-759.

13. van Dooijeweert C, van Diest PJ, Baas IO, et al. Variation in breast cancer grading: the effect of creating awareness through laboratory-specific and pathologist-specific feedback reports in 16734 patients with breast cancer. J Clin Pathol 2020. doi: 10.1136/jclinpath-2019-206362 